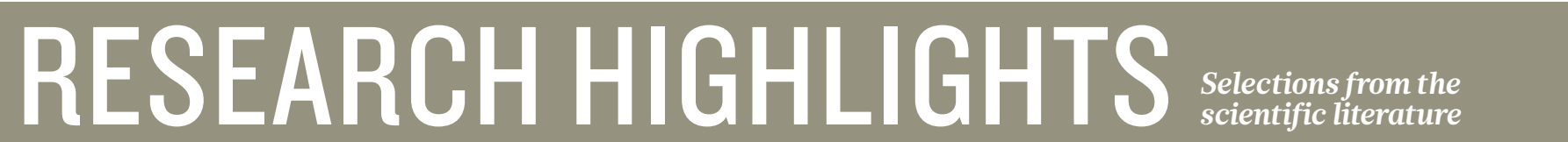

NEUROSCIENCE

\section{Cell junk hinders nerve regrowth}

Older animals recover slowly from nerve injury because they take longer to clear debris from damaged nerves than younger creatures do.

Hyuno Kang and Jeff Lichtman at Harvard University in Cambridge, Massachusetts, crushed the nerves that transmit signals to muscles in young and old mice, and used high-resolution time-lapse imaging to follow the nerves' regeneration.

Genetic engineering and staining techniques allowed the duo to distinguish cell types and cell debris by colour. The pair found that nerve growth slowed or temporarily halted when the tips of nerve fibres, or axons, hit lingering debris. Young mice broke down these nerve remnants faster than old mice did.

Axons in aged mice regenerated as quickly as those in young animals if their paths were clear of cell junk, so the authors suggest that nerveinjury therapies for older people could target debrisclearance mechanisms.

J. Neurosci. 33, 19480-19491 (2013)

ASTRONOMY

\section{Galactic clouds} swathed in fog

Molecular clouds, where stars are born, are surrounded by a fog of hydrogen that interacts with the clouds. These interactions could affect whether stars form.

In the Plateau de Bure Interferometer Arcsecond Whirlpool Survey, astronomers led by Eva Schinnerer of the Max Planck Institute for Astronomy in Heidelberg, Germany, mapped

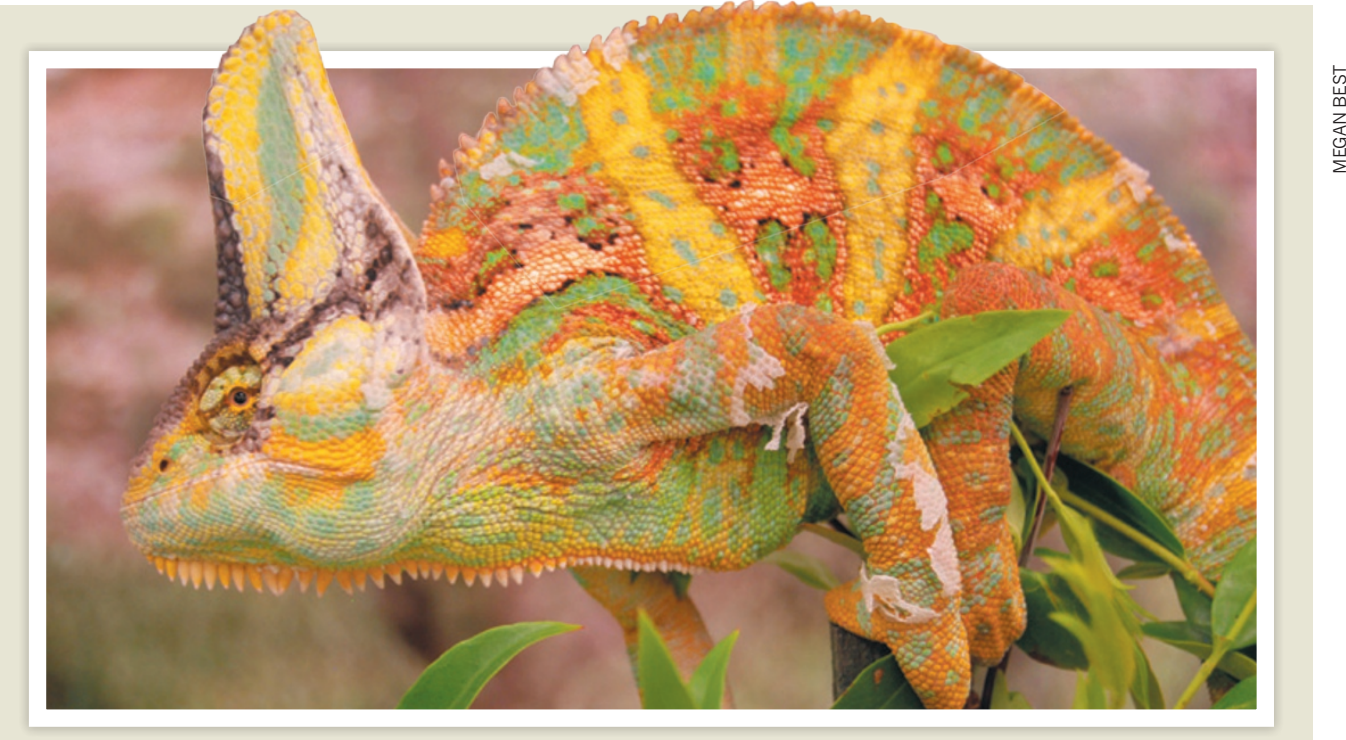

ANIMAL BEHAVIOUR

\section{Macho chameleons shine the brightest}

Male chameleons signal their aggressive intentions and prowess in fighting by adjusting the brightness of their skin colour.

Russell Ligon and Kevin McGraw at Arizona State University in Tempe staged fights between pairs of adult male veiled chameleons (Chamaeleo calyptratus; pictured) and used high-definition video to track how the animals' skin colour and brightness changed in 28 different patches on the body.
Individuals whose stripes brightened the most were more likely to charge another animal, while chameleons whose heads turned bright more quickly proved the most likely to win a bout.

The two signals could help male chameleons to separate a would-be combatant's motivation to fight from its ability to fight - two factors in competitive behaviour that can have different effects on the outcome of a contest. Biol. Lett. http://doi.org/qf9 (2013)
1,500 giant molecular clouds in the Whirlpool galaxy, about 7 million parsecs (23 million light years) away. They report in a series of papers that the clouds are not isolated as previously thought, but are embedded in a molecular fog that is much denser than expected and contains $50 \%$ of the galaxy's hydrogen.

Clouds that are surrounded by the fog experience decreased pressure when the fog moves relative to larger galactic structures, such as the arms of the spiral galaxy. This reduced pressure lowers the chances of star formation.

Astrophys. J. 779, 42; 43; 44; 45; 46 (2013)

\section{MOLECULAR BIOLOGY}

\section{Microbes meddle} with microRNA

Microbes in mouse guts can interfere with infection responses by changing the expression of small RNAs that regulate some genes.

Cristel Archambaud and Pascale Cossart at the Pasteur Institute in Paris and their colleagues looked at the expression of microRNA molecules in the small intestines of mice before and after infection with Listeria monocytogenes, a food-borne pathogen. They found reduced expression of six microRNAs after the infection in normal mice, but no decrease was seen for five of the microRNAs in mice without gut microbes, suggesting that the microbiota was responsible for this effect.

The findings suggest that gut microbes regulate the expression of protein-coding genes that are controlled by the microRNAs during infection. mBio 4, e00707-13 (2013)

\section{CANCER}

\section{Invasive tumours follow the leader}

Tumour cells that lead the charge in invasive breast cancer express two genes that are 